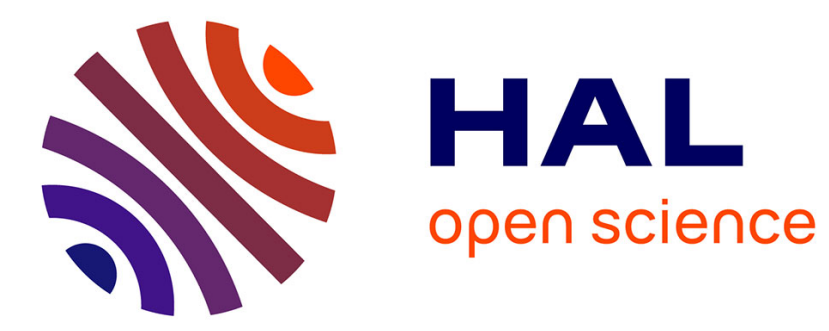

\title{
New limit cycles of dry friction oscillators under harmonic load
}

\author{
Madeleine Pascal
}

\section{To cite this version:}

Madeleine Pascal. New limit cycles of dry friction oscillators under harmonic load. Nonlinear Dynamics, 2012, 70 (2), pp.1435-1443. 10.1007/s11071-012-0545-5 . hal-00747517

\section{HAL Id: hal-00747517 \\ https://hal.science/hal-00747517}

Submitted on 6 Jun 2016

HAL is a multi-disciplinary open access archive for the deposit and dissemination of scientific research documents, whether they are published or not. The documents may come from teaching and research institutions in France or abroad, or from public or private research centers.
L'archive ouverte pluridisciplinaire HAL, est destinée au dépôt et à la diffusion de documents scientifiques de niveau recherche, publiés ou non, émanant des établissements d'enseignement et de recherche français ou étrangers, des laboratoires publics ou privés. 


\title{
New limit cycles of dry friction oscillators under harmonic load
}

\author{
Madeleine Pascal
}

\begin{abstract}
We consider a system composed of two masses connected by linear springs. One of the masses is in contact with a driving belt moving at a constant velocity. Friction force, with Coulomb's characteristics, acts between the mass and the belt. Moreover, the mass is also subjected to a harmonic external force. Several periodic orbits including stick phases and slip phases are obtained. In particular, the existence of periodic orbits including a part where the mass in contact with the belt moves in the same direction at a higher speed than the belt itself is proved. Non-sticking orbits are also found for a non-moving belt. We prove that this kind of solution is symmetric in space and in time.
\end{abstract}

Keywords Dry friction · Periodic orbits · Coupled oscillator $\cdot$ Stick-slip motion

\section{Introduction}

This paper is a continuation of several investigations [5-7], related to vibrating systems excited by dry friction. These systems are frequently encountered in many industrial applications. One of the most popular models of stick-slip oscillators consists of several masses connected by linear springs, one (or more) of the masses is in contact with a driving belt moving at a constant velocity. In the past, several authors investigated the behavior of this system, with different friction laws and with or without external actions and damping [1, 4], mainly via numerical approach.

However, assuming Coulomb's laws of dry friction, the corresponding dynamical model is a piecewise linear system, and even for multi-degree-of-freedom cases, some analytical results about the existence and the stability of periodic orbits including stick-slip phases have been obtained [5-7].

One interesting phenomenon is the existence, inside periodic orbits with stick and slip parts, of an "overshooting" slip phase. During this part of the orbit, the mass in contact with the belt moves in the same direction at a higher speed than the belt itself.

Up to now [9], this phenomenon has been observed only for more complex friction characteristics than Coulomb's ones. In [7], a self-excited stick-slip oscillator including two degrees of freedom is considered. Assuming Coulomb's laws of dry friction, a set of periodic orbits including an overshooting part is obtained using analytical methods.

In this work, we consider the same model of dry friction oscillator subjected to a harmonic external force. Several periodic orbits containing stick phases and slip phases are obtained. In particular, the existence of periodic orbits including an overshooting part is proved. 


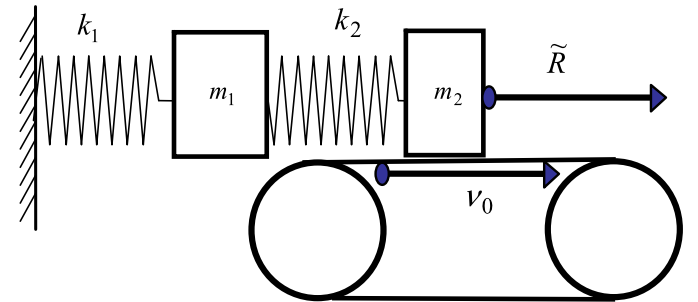

Fig. 1 Dry friction oscillator

\section{Problem formulation}

The system (Fig. 1) is composed of two masses $m_{1}, m_{2}$ connected by two linear springs of stiffness $k_{1}, k_{2}$. The second mass is in contact with a belt moving at a constant velocity $v_{0}$. A friction force $\tilde{F}$ acts between the mass and the belt. Moreover, the second mass is also subjected to an external force $\tilde{R}$ given by
$\tilde{R}=\tilde{p} \cos \left(\tilde{\omega} t^{\prime}+\varphi\right)$

( $\tilde{p}, \tilde{\omega}, \varphi$ are constant parameters)

The equations of motion related to this system are written as

$x_{1}^{\prime \prime}+x_{1}-\chi x_{2}=0$,

$x_{2}^{\prime \prime}+\chi \eta\left(x_{2}-x_{1}\right)=\eta u+p \cos (\omega t+\varphi)$

$x_{1}, x_{2}$ are the displacements of the masses,

$$
\begin{aligned}
& \eta=\frac{m_{1}}{m_{2}}, \quad \chi=\frac{k_{2}}{k_{1}+k_{2}}, \quad u=\frac{\tilde{F}}{k_{1}+k_{2}}, \\
& p=\eta \frac{\tilde{p}}{k_{1}+k_{2}}, \quad t=\Omega t^{\prime}, \quad \Omega=\sqrt{\frac{k_{1}+k_{2}}{m_{1}}}, \\
& (O)^{\prime}=\frac{d(O)}{d t}, \quad \omega=\frac{\tilde{\omega}}{\Omega}
\end{aligned}
$$

The dry friction force $u$ is obtained from Coulomb's laws:

$$
u= \begin{cases}u_{s} \operatorname{sign}\left(V-x_{2}^{\prime}\right), & \text { if } V-x_{2}^{\prime} \neq 0, \\ \chi\left(x_{2}-x_{1}\right)-\frac{p}{\eta} \cos (\omega t+\varphi), & \text { if } V-x_{2}^{\prime}=0,\left|\chi\left(x_{2}-x_{1}\right)-\frac{p}{\eta} \cos (\omega t+\varphi)\right|<u_{r}, \\ u_{s}, & \text { if } V-x_{2}^{\prime}=0, \quad \chi\left(x_{2}-x_{1}\right)-\frac{p}{\eta} \cos (\omega t+\varphi)>u_{r}, \\ -u_{s}, & \text { if } V-x_{2}^{\prime}=0, \chi\left(x_{2}-x_{1}\right)-\frac{p}{\eta} \cos (\omega t+\varphi)<-u_{r}, 0<u_{s}<u_{r}, V=\frac{\nu_{0}}{\Omega}\end{cases}
$$

$u_{r}$ is the friction force at rest (sticking), $u_{s}$ is the slipping friction force.

\section{Prediction of the oscillations exhibited by the system}

The dynamical behavior of this oscillator includes several phases of slip and stick motion of $m_{2}$. For each kind of motion, a close form solution is available.

\subsection{Slip motion of $m_{2}$ with $x_{2}^{\prime}<V$}

The solution is obtained from a modal analysis of (2) where $u=u_{s}$

$$
\begin{aligned}
& Z(t)=H(t)\left(Z_{0}-F_{0}\right)+F(t), \\
& F(t)=\left(\begin{array}{c}
R(t) \\
R^{\prime}(t)
\end{array}\right), \\
& R(t)=Q \cos (\omega t+\varphi), \quad F_{0}=F(0),
\end{aligned}
$$

$$
\begin{aligned}
& Z=\left(\begin{array}{c}
z \\
z^{\prime}
\end{array}\right), \quad Z_{0}=Z(0), \\
& z=X-d_{0}, \quad X=\left(x_{1}, x_{2}\right)^{t}, \\
& H(t)=\left(\begin{array}{cc}
H_{1}(t) & H_{2}(t) \\
H_{3}(t) & H_{1}(t)
\end{array}\right), \quad d_{0}=\left(d_{01}, d_{02}\right)^{t}, \\
& d_{01}=\frac{u_{s}}{1-\chi}, \quad d_{02}=\frac{d_{01}}{\chi}, \\
& Q=\left(q_{1}, q_{2}\right)^{t}, \quad q_{1}=\frac{p \chi}{\left(\omega^{2}-\omega_{1}^{2}\right)\left(\omega^{2}-\omega_{2}^{2}\right)}, \\
& q_{2}=q_{1} \frac{\left(1-\omega^{2}\right)}{\chi}
\end{aligned}
$$

The two by two matrices $H_{i}(t)(i=1,2,3)$ and the natural frequencies $\left(\omega_{1}, \omega_{2}\right)$ are obtained in analytical form (see Appendix 1).

\subsection{Slip motion of $m_{2}$ with $x_{2}^{\prime}>V$ (overshooting)}

The solution is obtained from (2) where $u=-u_{s}$

$$
\begin{aligned}
& Z(t)=H(t)\left(Z_{0}-F_{0}\right)+F(t)+2 L(t) d_{0}, \\
& L(t)=\left(\begin{array}{c}
H_{1}(t)-I \\
H_{3}(t)
\end{array}\right), \quad I=\left(\begin{array}{ll}
1 & 0 \\
0 & 1
\end{array}\right)
\end{aligned}
$$




\subsection{Stick motion of $m_{2}\left(x_{2}^{\prime}=V\right)$}

This motion is related to the following dynamical system:

$x_{1}^{\prime \prime}+x_{1}-\chi x_{2}=0, \quad x_{2}^{\prime \prime}=0$

The solution [5] is given by

$Z(t)=\Gamma(t) Z_{0}, \quad \Gamma(t)=\left(\begin{array}{ll}\Gamma_{1}(t) & \Gamma_{2}(t) \\ \Gamma_{3}(t) & \Gamma_{1}(t)\end{array}\right)$

The two by two matrices $\Gamma_{i}(t)(i=1,2,3)$ are given in Appendix 1. Moreover, during all this kind of motion, the following constraint holds:

$\left|\chi \eta\left(x_{2}-x_{1}\right)-p \cos (\omega t+\varphi)\right|<\eta u_{r}$

\section{Symmetrical periodic solutions}

A first set of periodic orbits of period $\Theta=2 \pi / \omega$ is obtained. These motions involve for each period first a slip motion of $m_{2}$ with $x_{2}^{\prime}<V$ followed by a phase of stick motion of the mass $\left(x_{2}^{\prime}=V\right)$. At the beginning $(t=0)$ of the slip motion, the initial conditions are given by

$z_{2}^{\prime}(0)=V$,

$\chi \eta\left(z_{2}(0)-z_{1}(0)\right)=p \cos \varphi+\eta\left(u_{r}-u_{s}\right)$

For $0<t<\tau$, the system undergoes a slip motion defined by (5).

At the end $(t=\tau)$ of the slip motion, the following condition is assumed:

$Z(\tau)=E Z_{0}, \quad E=\left(\begin{array}{cc}-I & 0 \\ 0 & I\end{array}\right)$

The time lag $\varphi$ of the external force is given by $\varphi=$ $(\pi-\omega \tau) / 2$. From (12) and (13), we deduce:

$$
\begin{aligned}
& -\eta\left(u_{r}+u_{s}\right)<\chi \eta\left(z_{2 c}-z_{1 c}\right)-p \cos (\omega \tau+\varphi) \\
& =-\eta\left(u_{r}-u_{s}\right)<\eta\left(u_{r}-u_{s}\right)
\end{aligned}
$$

For $\tau<t<\tau+T$, the system motion is a sticking motion given by

$$
Z(t)=\Gamma(t-\tau) E Z_{0}
$$

A periodic solution of period $\Theta$ is obtained if the following relation is fulfilled:

$Z_{0}=\Gamma(T) E Z_{0}, \quad(T=\Theta-\tau)$

Taking into account the properties (Appendix 1) of the matrices $H(t), \Gamma(t)$, the conditions for the existence of this periodic solution are given by the following system of four scalar equations:

$$
\begin{aligned}
& \left(H_{1}+I\right)\left(z_{0}-Q s_{0}\right)+H_{2}\left(z_{0}^{\prime}+Q \omega c_{0}\right)=0, \\
& \quad H_{i}=H_{i}(\tau) \\
& \left(\Gamma_{1}+I\right) z_{0}-\Gamma_{2} z_{0}^{\prime}=0, \quad \Gamma_{i}=\Gamma_{i}(T), \\
& \quad(i=1,2) \\
& s_{0}=\sin (\omega \tau / 2), \quad c_{0}=\cos (\omega \tau / 2)
\end{aligned}
$$

$\tau$ and hence the time duration $T=\Theta-\tau$ of the stick motion, together with the initial conditions $z(0), z^{\prime}(0)$ are computed. As in the case of the self-excited dry friction oscillator considered in [5], an interesting property of symmetry is proved for these orbits (see Appendix 2):

$$
\begin{aligned}
& Z(t)=E Z(\tau-t), \quad 0<t<\tau / 2, \quad \text { (slip motion) } \\
& Z\left(t_{1}\right)=E Z\left(T-t_{1}\right), \quad 0<t_{1}<T / 2,
\end{aligned}
$$

(stick motion), $\quad t_{1}=t-\tau$

A numerical validation is made for the following set of data:

$\chi=0.5, \quad \eta=3.8, \quad u_{s}=0.02, \quad u_{r}=0.1607$,

$V=1, \quad \omega=0.6, \quad p=0.05$

The other parameters related to this orbit are computed:

$\tau=7.749, \quad T=2.723, \quad \Theta=10.472$,

$z_{1}(0)=1.0609, \quad z_{2}(0)=1.3615, \quad z_{1}^{\prime}(0)=0.581$

The corresponding phase portraits $\left(z_{i}, z_{i}^{\prime}\right), i=(1,2)$ of the two masses are shown on Fig. 2: these curves are symmetrical with respect to the vertical line $z=0$. (The thick parts of the curves are related to the slip motion while the thin parts show the stick motion.)

The constraints derived from (11) during the stick motion:

$$
\begin{aligned}
\left(t<t_{1}<T, t_{1}=t-\tau\right) & \\
f_{1} \equiv & \chi \eta\left(z_{2}-z_{1}\right)+p \sin \left(\omega\left(t_{1}+\tau / 2\right)\right) \\
& \quad-\eta\left(u_{r}-u_{s}\right) \leq 0 \\
f_{2} \equiv & \chi \eta\left(z_{2}-z_{1}\right)+p \sin \left(\omega\left(t_{1}+\tau / 2\right)\right) \\
& +\eta\left(u_{r}+u_{s}\right)>0
\end{aligned}
$$

are fulfilled. 


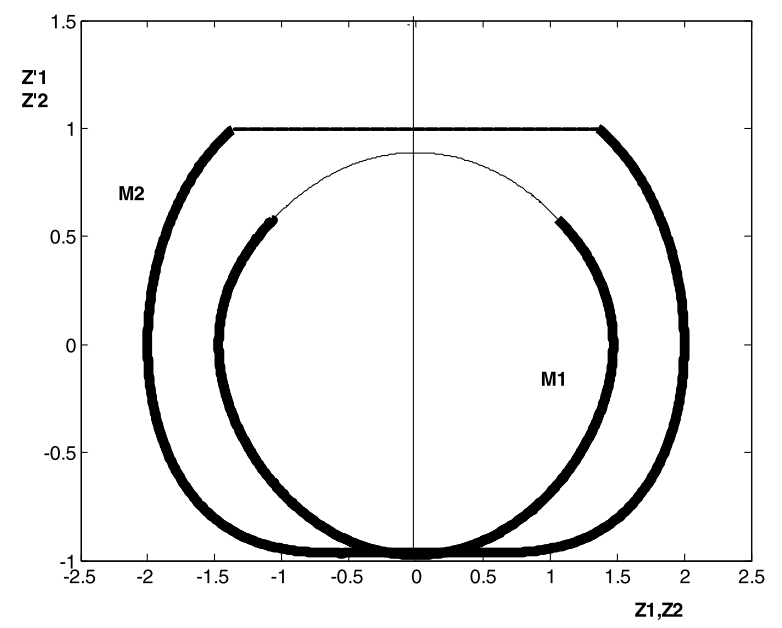

Fig. 2 Phase portraits of the symmetrical solution

\section{Periodic orbits including an overshooting part}

A second set of periodic orbits of period $\Theta=2 \pi / \omega$ is obtained. For each period, the motion is composed of three parts. The first one is a slip motion of $m_{2}$ with $x_{2}^{\prime}<V$ for $0<t<\tau$; the next part $\left(0<t-\tau<\tau_{1}\right)$ is an overshooting slip motion of the mass $\left(x_{2}^{\prime}>V\right)$; the last part $0<t-\tau-\tau_{1}<T$

$\left(T=\Theta-\tau-\tau_{1}\right)$ is a stick motion of $m_{2}$.

At the beginning of the motion for $t=0$, the conditions (12) are fulfilled. If at $t=\tau$, instead of (13), the following conditions:

$$
\begin{aligned}
& z_{2}^{\prime}(\tau)=V, \\
& \chi \eta\left(z_{2}(\tau)-z_{1}(\tau)\right)-p \cos (\omega \tau+\varphi) \\
& \quad+\eta\left(u_{r}+u_{s}\right)<0
\end{aligned}
$$

are fulfilled, we get an overshooting motion for $t>\tau$.

This motion ends at $t=\tau+\tau_{1}$ if, at this time:

$$
\begin{gathered}
z_{2 c}^{\prime} \equiv z_{2}^{\prime}\left(\tau+\tau_{1}\right)=V, \\
-\eta\left(u_{r}+u_{s}\right)< \\
\quad \chi\left(z_{2 c}-z_{1 c}\right) \\
-p \cos \left(\omega\left(\tau+\tau_{2}\right)+\varphi\right) \\
<\eta\left(u_{r}-u_{s}\right) \\
z_{i c} \equiv z_{i c}\left(\tau+\tau_{1}\right), \quad(i=1,2)
\end{gathered}
$$

For $\tau+\tau_{1}<t<\tau+\tau_{1}+T$, the system undergoes a sticking motion. A periodic solution of period $\tau+$ $\tau_{1}+T=\Theta$ is obtained if:

$$
Z(\Theta)=Z_{0}
$$

Taking into account the constraints deduced from (18), (19) and (20), the solution is defined by five linear equations with respect to $\left(z_{10}, z_{20}, z_{10}^{\prime}\right)$ :

$a_{i} z_{10}+b_{i} z_{20}+c_{i} z_{10}^{\prime}+d_{i}=0, \quad(i=1, \ldots, 5)$

$\left(a_{i}, b_{i}, c_{i}, d_{i}\right)$ are given in Appendix 3.

Assuming that $\Delta=\operatorname{det}\left(a_{j}, b_{j}, c_{j}\right) \neq 0$, $(j=1,2,3)$, the values of $\left(z_{10}, z_{20}, z_{10}^{\prime}\right)$ are obtained:

$$
z_{10}=\frac{\Delta_{1}}{\Delta}, \quad z_{20}=\frac{\Delta_{2}}{\Delta}, \quad z_{10}^{\prime}=\frac{\Delta_{3}}{\Delta}
$$$$
\Delta_{1}=\operatorname{det}-\left|\begin{array}{lll}
d_{1} & b_{1} & c_{1} \\
d_{2} & b_{2} & c_{2} \\
d_{3} & b_{3} & c_{3}
\end{array}\right|,
$$$$
\Delta_{2}=\operatorname{det}\left|\begin{array}{lll}
d_{1} & a_{1} & c_{1} \\
d_{2} & a_{2} & c_{2} \\
d_{3} & a_{3} & c_{3}
\end{array}\right|,
$$

$$
\Delta_{3}=\operatorname{det}\left|\begin{array}{lll}
d_{1} & b_{1} & a_{1} \\
d_{2} & b_{2} & a_{2} \\
d_{3} & b_{3} & a_{3}
\end{array}\right|
$$

The parameters $\left(\tau, \tau_{1}\right)$ are the roots of the compatibility conditions:

$$
\begin{aligned}
F_{k}\left(\tau, \tau_{1}\right) & \equiv a_{k+3} \Delta_{1}+b_{k+3} \Delta_{2}+c_{k+3} \Delta_{3}+d_{k+3} \Delta \\
& =0, \quad(k=1,2)
\end{aligned}
$$

Assuming that $\left(\chi, \eta, V, u_{s}\right)$ are given data, $u_{r}$ is deduced from the relation:

$u_{r}=u_{s}+f, \quad f=\chi\left(z_{20}-z_{10}\right)-\frac{p}{\eta} \cos \varphi$

An example of overshooting orbit is obtained for the values

$\chi=0.2, \quad \eta=4, \quad u_{s}=0.05, \quad V=1$,

$\omega=0.6289, \quad p=0.05, \quad \varphi=0$

The other parameters related to this solution are computed:

$\tau=4.909, \quad \tau_{1}=2.8275, \quad T=2.2543$,

$\Theta=9.9908, \quad u_{r}=0.4585, \quad z_{1}(0)=1.2809$,

$z_{2}(0)=3.3861, \quad z_{1}^{\prime}(0)=-0.4117$

The phase portraits $\left(z_{i}, z_{i}^{\prime}\right), i=(1,2)$ of the two masses are shown on Fig. 3. (The heavy thick part of 


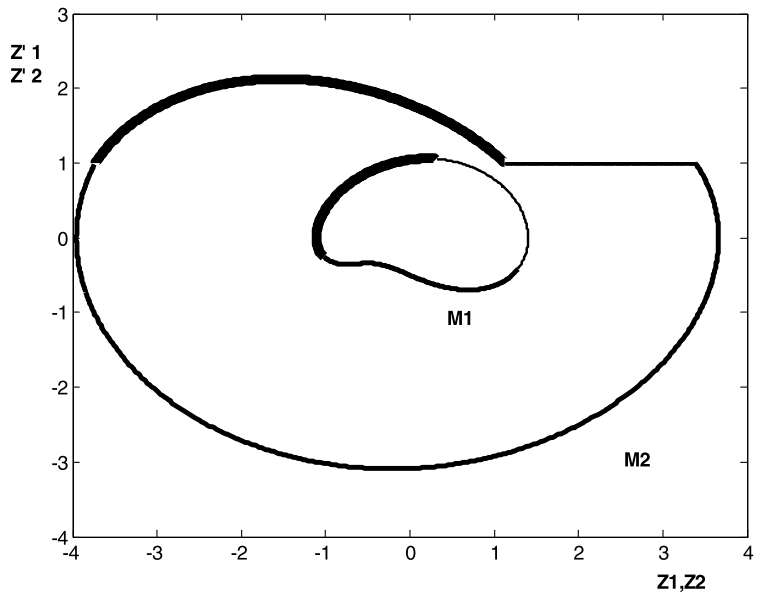

Fig. 3 Phase portraits of the overshooting periodic solution

the curves shows the overshooting motion while the thin one is related to the stick motion.)

\section{Non-sticking periodic solutions}

In industrial applications, avoiding sticking phases of motion is sometimes necessary. In the past, several authors $[2,3,8]$ investigated the existence of periodic non-sticking solutions of a one-degree-of-freedom oscillator subjected to simple harmonic loading. The mass is in contact with a fixed surface and a dry friction force acts between the mass and the surface. The aim of these works is to obtain some estimates about the minimum external force amplitude needed to prevent this sticking motion. The non-sticking orbit involves for each period a slipping motion with a negative mass velocity, and a slipping motion with a positive mass velocity (overshooting motion). Moreover, the authors assumed that the motion is symmetric in space $[2,3,8]$ and time $[3,8]$.

In the following, this problem is revisited for the two-degree-of-freedom oscillator considered in this work.

Let us consider the system described in Fig. 1, and assume the following initial conditions:

$z_{20}^{\prime}=V, \quad \chi \eta\left(z_{20}-z_{10}\right)>p \cos \varphi+\eta\left(u_{r}-u_{s}\right)$

For $0<t<\tau$, the system undergoes a slipping motion with $z_{2}^{\prime}<V$, given by (5).

This motion ends at $t=\tau$ if $z_{2 B}^{\prime} \equiv z_{2}^{\prime}(\tau)=V$.
Let us assume that $\tau=\pi / \omega=\Theta / 2$ and that

$$
\begin{aligned}
& \chi \eta\left(z_{2 B}-z_{1 B}\right)<p \cos (\omega \tau+\varphi)-\eta\left(u_{r}+u_{s}\right) \\
& \equiv-p \cos \varphi-\eta\left(u_{r}+u_{s}\right), \\
& z_{i B}=z_{i}(\tau), \quad(i=1,2)
\end{aligned}
$$

For $t>\tau$ the system undergoes an overshooting slipping motion $\left(z_{2}^{\prime}>V\right)$. This motion is given by

$$
\begin{aligned}
Z(t)= & H(t-\tau)\left(Z_{B}-F_{B}\right) \\
& +2 L(t-\tau) d_{0}+F(t)
\end{aligned}
$$

A periodic motion of period $\Theta=2 \pi / \omega$ is obtained if $Z_{0}=Z(\Theta) \equiv H(\tau)\left(Z_{B}-F_{B}\right)+2 L(\tau) d_{0}+F_{0}$,

$Z_{B}=Z(\tau)=H(\tau)\left(Z_{0}-F_{0}\right)+F_{B}$,

$F_{B}=F(\tau)=-F_{0}$

From (28) we deduce:

$[H(\tau)-H(-\tau)]\left[Z_{B}-F_{B}\right]+2 L(\tau) d_{0}=0$,

$H(\tau)-H(-\tau)=2\left|\begin{array}{cc}0 & H_{2} \\ H_{3} & 0\end{array}\right|$,

$H_{i}=H_{i}(\tau), \quad(i=1,2,3)$

From (29), we obtain

$$
\begin{aligned}
& H_{2}\left(z_{B}^{\prime}-Q \omega \sin \varphi\right)+\left(H_{1}-I\right) d_{0}=0 \\
& H_{3}\left(z_{B}+Q \cos \varphi+d_{0}\right)=0
\end{aligned}
$$

From (30), if $\operatorname{det}\left(H_{3}\right) \neq 0$, the following relations hold:

$z_{B}=-Q \cos \varphi-d_{0} \quad$ i.e. $x_{B}=-Q \cos \varphi$,

$z_{B}^{\prime}=Q \omega \sin \varphi+H_{2}^{-1}\left(I-H_{1}\right) d_{0}$

From (28), (31) and the relation:

$H_{1}^{2}-I=H_{2} H_{3}$, hence $H_{3}=H_{2}^{-1} H_{1}^{2}-H_{2}^{-1}$

the following results are obtained

$z_{0}=Q \cos \varphi-d_{0}, \quad$ i.e. $x_{0}=-x_{B}=Q \cos \varphi$

$z_{0}^{\prime}=-Q \omega \sin \varphi+H_{2}^{-1}\left(H_{1}-I\right) d_{0}=-z_{B}^{\prime}$

This last condition and the relation $z_{20}^{\prime} \equiv z_{2 B}^{\prime}=V$ lead to $V=0$.

In conclusion, non-sticking periodic orbits are obtained only for $V=0$, and the motion is symmetric in space and time (see Appendix 4).

The initial conditions and the time lag $\varphi$ of the external force are deduced from (33):

$$
\begin{aligned}
& z_{10}=q_{1} \cos \varphi-d_{01}, \quad z_{20}=q_{2} \cos \varphi-d_{02}, \\
& z_{10}^{\prime}=-q_{1} \omega \sin \varphi-a_{1} d_{01}-a_{2} d_{02} \\
& a_{1}=d\left(\varphi_{1} \lambda_{2}-\varphi_{2} \lambda_{1}\right), \quad a_{2}=d\left(\varphi_{2}-\varphi_{1}\right)
\end{aligned}
$$


$\sin \varphi=-\left(b_{1} d_{01}+b_{2} d_{02}\right) / q_{2} \omega$,

$\left|\left(b_{1} d_{01}+b_{2} d_{02}\right) / q_{2} \omega\right|<1$,

$b_{1}=d\left(\varphi_{1}-\varphi_{2}\right) \lambda_{1} \lambda_{2}, \quad b_{2}=d\left(\lambda_{2} \varphi_{2}-\lambda_{1} \varphi_{1}\right)$

$\varphi_{i}=\omega_{i} \tan g\left(\omega_{i} \pi / 2 \omega\right)$,

$\lambda_{i}=\frac{1-\omega_{i}^{2}}{\chi} \quad(i=1,2), \quad d=\chi /\left(\omega_{1}^{2}-\omega_{2}^{2}\right)$

The constraints deduced from (25) and (26) lead to the same condition:

$\chi \eta\left(q_{2}-q_{1}\right) \cos \varphi-p \cos \varphi-\eta u_{r}>0$

and from (36), a condition about the minimum value of the external force amplitude needed to avoid a sticking motion is obtained:

$p>\left|D \frac{b_{1} d_{01}+b_{2} d_{02}}{\left(1-\omega^{2}\right) \omega}\right|, \quad D=\left(\omega^{2}-\omega_{1}^{2}\right)\left(\omega^{2}-\omega_{2}^{2}\right)$

A numerical validation is performed for the following values of the parameters:

$\chi=0.3, \quad \eta=4, \quad \omega=0.6, \quad p=1$,

$u_{s}=0.1, \quad u_{r}=0.2996, \quad \Theta=10.472$

The corresponding values of the initial conditions and of the time lag $\varphi$ are obtained:

$x_{10}=1.5608, \quad x_{20}=3.3295$,

$x_{10}^{\prime}=0.1523, \varphi=0.3925$

The phase portraits $\left(x_{i}, x_{i}^{\prime}\right), i=(1,2)$ of the two masses are shown on Fig. 4 (the thick parts of the curves are related to the overshooting motion). These curves are symmetrical with respect to the origin 0 .

Under the assumption that there are only two transitions of motion during one period, we prove that nonsticking orbits are symmetric in space and time for almost all values of $\omega$ (see Appendix 5).

\section{Conclusion}

In this work, the steady state response of a two-degreeof-freedom oscillator subjected to dry friction and harmonic load is considered. Assuming Coulomb's laws of dry friction, the existence of several interesting periodic orbits, including stick and slip phases, is proved. In particular, periodic solutions with a phase during which the mass in contact with the belt moves faster

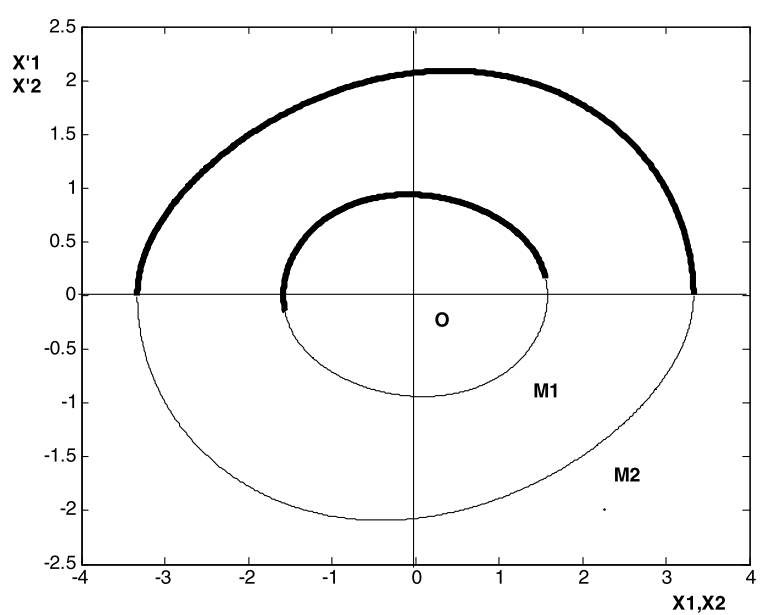

Fig. 4 Phase portrait of the non-sticking solution

than the belt are obtained. Moreover, in the case of a non-moving belt, a set of non-sticking periodic solutions is obtained, and we prove that these orbits are symmetrical in space and in time.

\section{Appendix 1}

$$
\begin{aligned}
& H_{i}(t)=\Lambda B_{i}(t) \Lambda^{-1}, \quad(i=1,2,3), \\
& B_{2}(t)=\left(\begin{array}{cc}
s_{1} / \omega_{1} & 0 \\
0 & s_{2} / \omega_{2}
\end{array}\right), \\
& s_{j}=\sin \left(\omega_{j} t\right)(j=1,2), \\
& B_{1}(t)=B_{2}^{\prime}(t), \quad B_{3}(t)=B /{ }^{\prime}(t)
\end{aligned}
$$

The natural frequencies $\left(\omega_{1}, \omega_{2}\right)$ are the roots of the characteristic equation:

$$
\begin{aligned}
& D\left(s^{2}\right) \equiv \operatorname{det}\left(K-I s^{2}\right)=0, \\
& K=\left(\begin{array}{cc}
1 & -\chi \\
-\chi \eta & \chi \eta
\end{array}\right)
\end{aligned}
$$

The eigenvectors $\psi_{j}=\left(\begin{array}{c}1 \\ \lambda_{j}\end{array}\right),(j=1,2)$ are defined by $\left(K-I \omega_{j}^{2}\right) \psi_{j}=0$.

These matrices fulfil the following property:

$$
\begin{aligned}
& H_{1}^{2}(t)-H_{2}(t) H_{3}(t)=0, \\
& \Gamma_{i}(t)=\Sigma \gamma_{i}(t) \Sigma^{-1}, \quad(i=1,2,3), \\
& \gamma_{2}(t)=\left(\begin{array}{cc}
\sin t & 0 \\
0 & t
\end{array}\right), \quad \Sigma=\left(\begin{array}{cc}
1 & \chi \\
0 & 1
\end{array}\right), \\
& \gamma_{1}(t)=\gamma_{2}^{\prime}(t), \quad \gamma_{3}(t)=\gamma^{\prime \prime} 2(t)
\end{aligned}
$$

The matrices $\Gamma_{i}(t)$ fulfil also the property:

$\Gamma_{1}^{2}(t)-\Gamma_{2}(t) \Gamma_{3}(t)=0$ 


\section{Appendix 2}

For $\tau / 2<t<\tau$, the periodic solution is defined by

$Z(\tau-t)=H(\tau-t)\left(Z_{0}-F_{0}\right)+F(\tau-t)$

From the identities:

$$
\begin{aligned}
& F(\tau-t)=E F(t), \quad\left(\text { i.e. } F(\tau)=E F_{0}\right) \\
& H(\tau-t)=H(\tau) H(-t), \quad H(-t) E=E H(t)
\end{aligned}
$$

the first relation (17) is deduced.

For $T / 2<t_{1}<T$, the solution is defined by

$$
Z(t)=\Gamma\left(T-t_{1}\right) E Z_{0}
$$

From the identities:

$$
\begin{aligned}
& \Gamma\left(T-t_{1}\right)=\Gamma(T) \Gamma\left(-t_{1}\right), \\
& E \Gamma\left(-t_{1}\right)=\Gamma\left(t_{1}\right) E, \\
& Z(\Theta)=\Gamma(T) E Z_{0}=Z_{0}
\end{aligned}
$$

the last relation (17) follows.

\section{Appendix 3}

$$
\begin{aligned}
a_{1}= & \tilde{H}_{11}-\Gamma_{11}, \quad b_{1}=\tilde{H}_{12}-\Gamma_{12} \\
c_{1}= & \tilde{H}_{13}-\Gamma_{13} \\
d_{1}= & -\left(\tilde{H}_{11} q_{1}+\tilde{H}_{12} q_{2}\right) \cos \varphi \\
& +\omega\left(\tilde{H}_{13} q_{1}+\tilde{H}_{14} q_{2}\right) \sin \varphi+q_{1} \cos \varphi_{C} \\
& +2\left(h_{11}-1\right) d_{01}+2 h_{12} d_{02}+\left(\tilde{H}_{14}+\Gamma_{14}\right) V \\
a_{2}= & \tilde{H}_{21}, \quad b_{2}=\tilde{H}_{22}-1, \quad c_{2}=\tilde{H}_{23} \\
d_{2}= & -\left(\tilde{H}_{21} q_{1}+\tilde{H}_{22} q_{2}\right) \cos \varphi \\
& +\omega\left(\tilde{H}_{23} q_{1}+\tilde{H}_{24} q_{2}\right) \sin \varphi+q_{2} \cos \varphi_{C} \\
& +2 h_{21} d_{01}+2\left(h_{22}-1\right) d_{02}+\left(\tilde{H}_{24}+T\right) V \\
a_{3}= & \tilde{H}_{31}+\Gamma_{31}, \quad b_{3}=\tilde{H}_{32}+\Gamma_{32} \\
c_{3}= & \tilde{H}_{11}-\Gamma_{11} \\
d_{3}= & -\left(\tilde{H}_{31} q_{1}+\tilde{H}_{32} q_{2}\right) \cos \varphi \\
& +\omega\left(\tilde{H}_{11} q_{1}+\tilde{H}_{12} q_{2}\right) \sin \varphi-q_{1} \sin \varphi_{C} \\
& +2 h_{31} d_{01}+2 h_{32} d_{02}+\left(\tilde{H}_{12}-\Gamma_{12}\right) V \\
\quad b_{4}=H_{42}, & c_{4}=H_{21}, \\
a_{4}= & H_{41}, \\
d_{4}= & \left(H_{22}-1\right) V-q_{2} \omega \sin \left(\varphi_{B}\right) \\
& -\left(H_{41} q_{1}+H_{42} q_{2}\right) \cos \varphi \\
& +\left(H_{21} q_{1}+H_{22} q_{2}\right) \omega \sin \varphi \\
&
\end{aligned}
$$


From (56), we deduce:

$$
\begin{aligned}
\xi_{1} & \equiv Z_{B}-F_{B}+E\left(Z_{0}-F_{0}\right) \\
& =(H(\tau)+E)\left(Z_{0}-F_{0}\right), \\
\xi_{2} & \equiv \bar{Z}_{B}-F_{B}+E\left(\bar{Z}_{0}-F_{0}\right) \\
& =\left(H\left(-\tau_{1}\right)+E\right)\left(\bar{Z}_{0}-F_{0}\right)
\end{aligned}
$$

From the relations:

$$
H(t) E=E(H(t))^{-1}=E H(-t)
$$

it follows:

$$
\begin{aligned}
& (H(-t)-E)(H(t)+E)=0, \\
& (H(t)-E)(H(-t)+E)=0
\end{aligned}
$$

From (57) and (59), we deduce

$$
(H(-\tau)-E) \xi_{1}=0, \quad\left(H\left(\tau_{1}\right)-E\right) \xi_{2}=0
$$

On the other hand, it is not difficult to show that

$\xi_{1}-\xi_{2}=Z_{B}-\bar{Z}_{B}+E\left(Z_{0}-\bar{Z}_{0}\right)=0$

Let us introduce the following notations:

$$
\begin{aligned}
\xi_{i}= & \left(\begin{array}{c}
X_{i} \\
Y_{i}
\end{array}\right), \quad(i=1,2) \\
X_{1}= & \left(H_{1}-I\right)\left(z_{0}-Q \cos \varphi\right) \\
& +H_{2}\left(z_{0}^{\prime}+Q \omega \sin \varphi\right), \\
Y_{1}= & H_{3}\left(z_{0}-Q \cos \varphi\right) \\
& +\left(H_{1}+I\right)\left(z_{0}^{\prime}+Q \omega \sin \varphi\right), \\
X_{2}= & \left(h_{1}-I\right)\left(\bar{z}_{0}-Q \cos \varphi\right) \\
& -h_{2}\left(z_{0}^{\prime}+Q \omega \sin \varphi\right), \\
Y_{2}= & -h_{3}\left(\bar{z}_{0}-Q \cos \varphi\right) \\
& +\left(h_{1}+I\right)\left(z_{0}^{\prime}+Q \omega \sin \varphi\right), \\
H_{j}= & H_{j}(\tau), h_{j}=h_{j}\left(\tau_{1}\right), \quad(j=1,2,3)
\end{aligned}
$$

From (60), it results:

$$
\begin{array}{ll}
Y_{1}=P_{1} X_{1}, & P_{1}=H_{2}^{-1}\left(H_{1}+I\right), \\
Y_{2}=P_{2} X_{2}, & P_{2}=-h_{2}^{-1}\left(h_{1}+I\right)
\end{array}
$$

The relations $X_{1}=X_{2}, Y_{1}=Y_{2}$, deduced from (61) are equivalent to:

$X_{1}=X_{2}, \quad P X_{1}=0, \quad P=P_{1}-P_{2}$

From (64), two cases are obtained:

1. $\operatorname{Det}(P) \neq 0$ leads to $X_{1}=0$, hence

$$
X_{2}=0, \quad Y_{1}=0, \quad Y_{2}=0
$$

From (56) and (64), we deduce

$$
\begin{aligned}
& z_{B}-Q \cos \varphi_{B}=z_{0}-Q \cos \varphi, \\
& z_{B}^{\prime}+Q \omega \sin \varphi_{B}=-\left(z_{0}^{\prime}+Q \omega \sin \varphi\right), \\
& \bar{z}_{B}-Q \cos \varphi_{B}=\bar{z}_{0}-Q \cos \varphi
\end{aligned}
$$

From the relation:

$z_{B}^{\prime}=-Q \omega \sin \varphi_{B}-\left(z_{0}^{\prime}+Q \omega \sin \varphi\right)$,

$z_{2 B}^{\prime}=z_{20}^{\prime}=0$

we obtain $\sin \varphi_{B}=-\sin \varphi$, hence

$\varphi_{B} \equiv \omega \tau+\varphi=-\varphi, \quad \varphi=-\omega \tau / 2 \quad$ or $\varphi_{B} \equiv \omega \tau+\varphi=\pi+\varphi, \quad$ hence $\tau=\pi / \omega=\tau_{1}$

In the first case, $\left(\varphi_{B} \equiv-\varphi, \varphi=-\omega \tau / 2\right)$, from (67) $z_{B}^{\prime}=-z_{0}^{\prime}$ and from (66):

$z_{B}=z_{0}-Q \cos \varphi+Q \cos \varphi_{B}=z_{0}$

which is impossible because for $0<t<\tau, z_{2}^{\prime}<0$, hence: $z_{2 B}<z_{20}$.

In the second case, $\left(\tau=\pi / \omega=\tau_{1}\right)$, hence $H_{i}=$ $h_{i}, i=1,2,3$

$z_{B}+Q \cos \varphi=z_{0}-Q \cos \varphi, \quad$ i.e.,

$x_{B}+Q \cos \varphi=x_{0}-Q \cos \varphi$.

From (56), we get

$$
\begin{aligned}
& z_{B}+\bar{z}_{B}+2 Q \cos \varphi \\
& \quad=H_{1}\left(z_{0}+\bar{z}_{0}-2 Q \cos \varphi\right) \quad \text { i.e. } \\
& x_{B}+Q \cos \varphi \\
& \quad=H_{1}\left(x_{0}-Q \cos \varphi\right)=x_{0}-Q \cos \varphi
\end{aligned}
$$

Hence, if $\operatorname{det}\left(H_{1}-I\right) \neq 0$, we obtain

$$
x_{0}=Q \cos \varphi, \quad x_{B}=-Q \cos \varphi=-x_{0}
$$

2. $\operatorname{Det}(P)=0$

From $P=H_{2}^{-1}\left(H_{1}+I\right)+h_{2}^{-1}\left(h_{1}+I\right)$ : we deduce

$$
\begin{aligned}
& P=\Lambda \tilde{P} \Lambda^{-1}, \quad \tilde{P}=\left(\begin{array}{cc}
\rho_{1} & 0 \\
0 & \rho_{2}
\end{array}\right), \\
& \Lambda=\left(\begin{array}{cc}
1 & 1 \\
\lambda_{1} & \lambda_{2}
\end{array}\right) \\
& \rho_{i}=\omega_{i} \frac{\sin \left(\alpha_{i}+\beta_{i}\right)}{\left(\sin \alpha_{i}\right)\left(\sin \beta_{i}\right)}, \quad(i=1,2) \\
& \alpha_{i}=\omega_{i} \tau / 2, \quad \beta_{i}=\omega_{i} \tau_{1} / 2
\end{aligned}
$$

It results $\operatorname{Det}(P)=0$ if $\sin \left(\alpha_{i}+\beta_{i}\right) \equiv$ $\sin \left(\omega_{i} \pi / \omega\right)=0$, i.e., $\omega_{i}=k \omega,(k=1,2, \ldots)$ (resonance). 
Except this particular case of resonance with the natural frequencies of the system, only symmetrical periodic solutions with a phase of slipping motion and a phase of overshooting motion exist.

\section{References}

1. Andreaus, U., Casini, P.: Dynamics of friction oscillators excited by a moving base and/or driving force. J. Sound Vib. 245(4), 685-699 (2001)

2. Csernak, G., Stepan, G.: On the periodic response of a harmonically excited dry friction oscillator. J. Sound Vib. 295(4), 649-658 (2006)

3. Hong, H.K., Liu, C.S.: Non-sticking oscillation formulae for Coulomb friction under harmonic loading. J. Sound Vib. 244(5), 883-898 (2001)
4. Galvanetto, U., Bishop, S.R.: Stick-slip vibrations of a 2degree-of-freedom geophysical fault model. Int. J. Mech. Sci. 36(8), 683-698 (1994)

5. Pascal, M.: Dynamics of coupled oscillators excited by dry friction. J. Comput. Nonlinear Dyn. 3(3), 20-26 (2008)

6. Pascal, M.: Two models of non smooth dynamical systems. Int. J. Bifurc. Chaos 21(10), 2853-2860 (2011)

7. Pascal, M.: New events in stick-slip oscillators behaviour. J. Appl. Math. Mech. 75(3), 402-409 (2011)

8. Shaw, S.W.: On the dynamic response of a system with dry friction. J. Sound Vib. 108(2), 305-325 (1986)

9. Teufel, A., Steindl, A., Troger, H.: On non smooth bifurcations in a simple friction oscillator. PAMM 5, 139-140 (2005) 